\title{
ALGUNOS ASPECTOS METODOLÓGICOS DE LA INVESTIGACIÓN EN RESOLUCIÓN DE PROBLEMAS EN FÍSICA: UNA REVISIÓN *
}

\author{
Some methodological issues \\ involved in Physics problems solving: a review
}

Laura Buteler ${ }^{1}$

Zulma Gangoso ${ }^{2}$

\begin{abstract}
Resumen: El trabajo presenta una revisión de los aspectos metodológicos involucrados en el estudio de las representaciones mentales puestas en juego por las personas para realizar ciertas tareas cognitivas. La revisión se acota al ámbito de la investigación en enseñanza de las ciencias y en particular, a un conjunto de investigaciones que abordan tareas estrechamente relacionadas con la resolución de problemas en Física. La revisión permite analizar y discutir los alcances y limitaciones de las decisiones metodológicas usuales para el estudio de la resolución de problemas en Física. Se ponen en consideración algunas directrices metodológicas plausibles de ser tenidas en cuenta para esta tarea.

Palabras clave: Representaciones. Resolución de problemas en Física. Métodos de indagación. Protocolos verbales.
\end{abstract}

\begin{abstract}
This work presents a review of the methodological issues involved in the studies of the representations used by people carrying out cognitive tasks. The review is based on literature dealing with cognitive tasks related to Physics problem solving. This review allows the analysis and discussion of the advantages and disadvantages of the usual methodological decisions in Physics problem solving. Methodological directions for physics problem solving are considered.
\end{abstract}

Key words: Representations. Physic problem solving. Methodology. Verbal protocols.

\footnotetext{
* Una discusión preliminar se presentó en la XIII Reunión Nacional de Profesores de Física en Río Cuarto, Córdoba (Argentina), en noviembre de 2003.

${ }^{1}$ Doctor en Física; docente, Facultad de Matemática, Astronomía y Física (FaMAF), Universidad Nacional de Córdoba, Argentina (UNC); Grupo de Investigación de Enseñanza de la Ciencia y la Tecnología (GECyT); Consejo Nacional de Investigaciones Científicas y Técnicas (CONICET).<lbuteler@famaf.unc.edu.ar>

${ }^{2}$ Doctor en Física; docente, FaMAF, UNC; (GECyT).<zulma@famaf.unc.edu.ar2>.
}

\footnotetext{
${ }^{1}$ Haya de la Torre esquina Medina Allende

Ciudad Universitaria

Córdoba 5000, Argentina
} 


\section{Introducción}

En Psicología Cognitiva las fuentes de evidencias experimentales constituyen valiosos elementos para poner a prueba las teorías o modelos que intentan explicar ciertos fenómenos cognitivos (CLEMENT, 2000). Por esta razón, como en toda ciencia, los procedimientos para obtener e interpretar esos datos no pueden ser discutidos en términos libres de teoría. Ericsson y Simon (1981) abordaron en detalle esta problemática:

La cuestión epistemológica (cómo el investigador obtiene información confiable acerca del sujeto) está íntimamente ligada con la cuestión psicológica (cómo el sujeto obtiene información confiable acerca del mundo). Como resultado, los psicólogos que han mantenido diferentes posiciones teóricas acerca de las leyes sustantivas de la psicología, han mantenido también diferentes posiciones con respecto a la validez de los distintos tipos de evidencias experimentales, y vice versa. (p. 16)

Si los procesos cognitivos son pensados en términos de representaciones y procedimientos que actúan sobre ellas, los métodos utilizados para testear teorías, pueden, en principio, dividirse en dos clases que obedecen a distintas perspectivas. Cada una de ellas da origen a procedimientos alternativos y complementarios.

Una clase contiene a los programas computacionales que describen (en lenguajes formales apropiados) las teorías, o ciertos aspectos de ellas, en términos de procedimientos algorítmicos actuando sobre una base de datos. Si bien estos programas que simulan comportamientos son "teorías" en sí mismos ${ }^{3}$ los resultados de la simulación constituyen una herramienta metodológica poderosa para validar si la teoría o el modelo propuesto, es efectivamente computable y auto consistente. Se trata de una prueba de consistencia interna del modelo. Sin embargo, si lo que se pretende simular es la cognición humana, el procedimiento anterior es largamente insuficiente. Desde esta perspectiva, la Psicología Cognitiva requiere además que los resultados de la simulación sean consistentes con los datos que arroja la experimentación con sujetos cuando éstos realizan las mismas tareas que aquellas para las cuales el programa ha sido construido. Esta necesidad da lugar a la segunda perspectiva: el conjunto de métodos que permite arrojar datos acerca de los procesos cognitivos humanos y que están relacionados con la consistencia externa o la plausibilidad psicológica del modelo propuesto.

En este trabajo se revisan cuestiones metodológicas involucradas en la segunda perspectiva, es decir, a la obtención de datos a partir de la actuación de sujetos puestos a realizar ciertas tareas cognitivas. La revisión se acota a dos líneas de investigación que han tenido y tienen aún hoy alto impacto en el ámbito de la enseñanza de las ciencias, y que resultan de particular interés para la tarea de resolución de problemas en Física.

\footnotetext{
${ }^{3}$ Un análisis más detallado de los distintos niveles teóricos acerca de la cognición desde esta perspectiva, se encuentra en Thagard (1996).
} 
Algunos aspectos metodológicos de la investigación...

Una de ellas es la línea de investigación de expertos y novatos en Física que caracteriza el conocimiento y las estrategias puestas en juego por sujetos expertos y novatos al resolver problemas de Física. Estas investigaciones se seleccionan para el análisis metodológico porque constituyen un referente ineludible en el estudio de la resolución de problemas en física desde un enfoque cognitivo. La otra línea de investigación se refiere a los estudios sobre modelos mentales. Esta propuesta intenta dilucidar la naturaleza de las representaciones mentales que las personas construyen ante ciertas situaciones particulares. El interés de este enfoque para la resolución de problemas en Física radica en que el primer estadio del proceso de solución consiste en la construcción de una representación interna de la situación que plantea el problema. Esta representación guía el proceso de solución y se construye a partir de lo que el sujeto sabe. Resulta plausible pensar a esta representación dinámica en términos de un modelo mental.

A partir de la revisión y el análisis de los aspectos metodológicos de las investigaciones referidas, se proponen algunos criterios que permitan diseñar situaciones experimentales útiles para obtener datos válidos en el ámbito de la resolución de problemas en Física.

\section{Una revisión metodológica}

\section{Los estudios de expertos y novatos en resolución de problemas de Física}

El nacimiento de estos estudios, ligado al origen de la Psicología Cognitiva y la Inteligencia Artificial en el marco de la Ciencia Cognitiva, provee los primeros resultados empíricos en el ámbito de la resolución de problemas en Física. La mayoría de estos trabajos, aunque exploratorios y considerados como fuente de datos para posibles y futuras simulaciones, mantienen el supuesto de un modelo cognitivo constituido por representaciones conteniendo datos y procesos que actúan sobre ellas. Se han seleccionado dos artículos considerados representativos para mostrar algunas características teóricas y metodológicas de esta línea de investigación.

Un estudio clásico de expertos y novatos en física, es el trabajo de Chi, Feltovich y Glaser (1981), acerca de la categorización y la representación de problemas de física por sujetos expertos y novatos. Para ellos, la representación interna de un problema de física es construida por el sujeto que resuelve sobre la base de su conocimiento específico (y su organización) en ese dominio. La hipótesis concreta de trabajo es que la representación es construida en el contexto de conocimiento disponible para problemas "tipo", y que ese conocimiento es "indicado" (en el sentido de ser localizado o ubicado en un índice de entrada) cada vez que un problema de física es colocado en una categoría. Así, las diferencias entre expertos y novatos en las categorizaciones de los problemas de física, estarían relacionadas con la estructura y el contenido de sus representaciones de diferentes problemas "tipo". A partir del concepto de esquema en el sentido de Rumelhart ${ }^{4}$, identifican las categorías impuestas por los sujetos sobre

\footnotetext{
${ }^{4}$ Un esquema es un formato representacional que contiene información prototípica o genérica y un conjunto de variables cuyos valores son inferidos por defecto cada vez ante una situación particular. Son usualmente utilizados para representar conceptos o estructuras conceptuales. Ver por ej. Pozo (1989).
} 
los problemas con esquemas de problemas tipo, dejando explícita la relación entre las decisiones metodológicas (categorizar problemas) con los conceptos teóricos involucrados.

Bajo el supuesto de que durante el proceso de categorización se activan los esquemas pertinentes a esas categorías, los investigadores exploran el contenido de esos esquemas y el proceso de activación de los mismos. La consigna dada a estos sujetos fue que dijeran en tres minutos todo lo que pudieran acerca de los problemas representantes de cada categoría, y que verbalizaran los potenciales caminos de solución. El análisis que realizan los autores a partir de las verbalizaciones consiste en convertir a los protocolos en reglas de producción ${ }^{5}$, permitiendo ver la dinámica de la representación. Las reglas de producción son estructuras de condiciones y acciones, tipo si-entonces o si-cuando. Esta decisión metodológica obedece al modelo cognitivo subyacente que consiste en postular no sólo representaciones (que son interpretadas en términos de esquemas de problemas), sino también de procesos que actúan sobre ella. Así infieren que algunas de esas producciones "activan" los esquemas que les permiten resolver los problemas.

La descripción anterior, aunque lejos de ser completa, revela algunos aspectos metodológicos interesantes. En primer lugar, queda clara la relación entre los conceptos teóricos y operacionales involucrados en las conjeturas iniciales del estudio (la correspondencia entre esquemas y categorización de problemas) con la interpretación que los autores realizan de los datos recogidos, lo cual revela explícitamente la conexión entre el dominio teórico y las evidencias empíricas. Por otra parte, cada vez que interesa recabar información relacionada con procesos, los datos considerados válidos son los generados a partir de las verbalizaciones espontáneas de los sujetos mientras resuelven los problemas.

En segundo lugar, como resultado de algunas de las actividades planteadas, los protocolos verbales de los novatos resultan casi imposibles de analizar en los mismos términos que los de los expertos. Estos protocolos resultan incompletos para la información que se desea extraer de ellos. Por ejemplo, en el caso de los análisis de los protocolos en términos de reglas de producción, los novatos verbalizan un gran número de reglas de producción sin consecuente, es decir, de "reglas incompletas".

Otro ejemplo representativo de este conjunto de estudios, es el de Larkin (1983). El trabajo propone un estadio característico en el proceso de solución para los expertos, que consiste en la construcción de una representación física que guía la solución. Este estadio, generalmente ausente en los novatos, consiste en una representación que contiene los principios físicos fundamentales que son necesarios para resolver los problemas planteados. La característica compartida por expertos y novatos sería la representación ingenua ("naive" en su idioma original) que contiene elementos concretos de esa situación, cuyas propiedades son difusas (mayoritariamente dependientes de variables contextuales), y que evoluciona en tiempo real.

\footnotetext{
${ }^{5}$ La flexibilidad de las reglas de producción como formato representacional, ha hecho de ellas un elemento indispensable en el ámbito de la inteligencia artificial. De hecho, los programas computacionales utilizan, en última instancia, reglas como forma para representar el conocimiento. Dado el origen del campo de investigación al que pertenece este estudio (experticia humana en la resolución de problemas), es plausible pensar en un análisis de este tipo.
} 
Algunos aspectos metodológicos de la investigación...

Basada en trabajos previos sobre expertos y novatos, Larkin (1983) supone que las fuentes de las representaciones físicas son esquemas (en el mismo sentido del trabajo de CHI, FELTOVICH y GLASER antes discutido) de los distintos principios físicos relevantes a cada problema. Para cada esquema, propone reglas de construcción y reglas de extensión que permiten hacer inferencias y llenar los "huecos" o casilleros vacíos en el esquema, generando nueva información. Las reglas de construcción, actúan sobre la representación ingenua y producen entidades que forman parte de la representación física, mientras que las reglas de extensión, actúan sobre la representación física agregando nuevas entidades en esa representación.

Uno de los problemas planteados consiste en calcular la velocidad que alcanza un cuerpo en la base de un plano inclinado, dejado en libertad en el extremo superior del mismo, suponiendo que existe rozamiento entre ambas superficies. Los esquemas posibles de ser utilizados en este caso, son el esquema correspondiente a la segunda ley de Newton, o bien el esquema correspondiente al teorema del trabajo y la energía. Si se utilizara el primer esquema, los huecos son rellenados con las fuerzas que actúan sobre el objeto

$\mathrm{F}_{\mathrm{g}}=\mathrm{mg} \operatorname{sen} \theta$ y $\mathrm{f}=\mu \mathrm{mg} \cos \theta$ (vía las reglas de construcción),

y la aceleración $\mathrm{a}=v^{2} / 2 \mathrm{l}$ (vía reglas de extensión),

donde $l$ es la longitud del plano inclinado, $\theta$ el ángulo de inclinación,

y $v$ la velocidad del cuerpo cuando llega a la base del plano.

Para poner a prueba su propuesta, Larkin (1983) toma como registros, los protocolos verbales de los sujetos, mientras resuelven cada problema. Las instrucciones dadas son que "piensen en voz alta" durante la resolución. Las unidades de información derivadas de los esquemas relevantes (como las dos fuerzas y la aceleración en el esquema de la segunda ley de Newton del ejemplo anterior), son identificadas en los protocolos de los sujetos expertos, así como el orden en que las verbalizan. Recién a partir de este "relleno", los expertos proceden a escribir otras ecuaciones matemáticas para calcular el valor requerido.

En el caso de los protocolos de los novatos, la generación de las ecuaciones para obtener el valor requerido ocupa el primer lugar, dejando para el último, las unidades de información que, en los expertos, se derivan de los esquemas de los principios físicos. En todos los casos, la verbalización de los productos de las inferencias que se supone que el individuo ejecuta, y el lugar que ocupan en la verbalización, constituyen las evidencias empíricas de su propuesta, quedando suficientemente clara la relación entre las hipótesis sustantivas del trabajo, las definiciones operacionales y los datos.

Por último, aparece en este estudio, una característica ya advertida en la primera investigación presentada en este apartado. Sólo 7 de los 11 registros pertenecientes a los sujetos novatos fueron interpretados. Si bien no se presentan detalles específicos, la autora excluye los cuatro protocolos restantes por considerarlos "no interpretables". Si se entiende por "no interpretable" aquel protocolo que contiene datos empíricos que no se ajustan al modelo teórico propuesto, entonces es posible inferir que la actuación de estos novatos no puede explicarse a partir del modelo propuesto. Más allá del valor indiscutible de los trabajos antes 
descriptos, se advierte la necesidad de incorporar elementos teóricos que permitan describir y/o explicar el comportamiento de los sujetos novatos. Los estudios sobre modelos mentales intentan producir resultados en esa dirección.

\section{Los estudios sobre modelos mentales}

Un conjunto de investigaciones desarrolladas en ámbitos relacionados con la instrucción formal en física, ha incorporado el constructo modelo mental como formato representacional del conocimiento (TREAGUST, CHITTLEBOROUGH y MAMIALA, 2004; TABER, 2003; BUCKLEY y BOULTER, 2000; KRAPAS, ALVES y CARVALHO, 2000; BORGES, 1998; DIAKIDOY, VOSNIADOU y HAWKS, 1997; GRECA y MALLMANN, 1997; GRECA y MOREIRA, 1997; HALLOUN, 1996; HARRISON y TREAGUST, 1996; VOSNIADOU, 1994; GUTIERREZ y OGBORN, 1992; COLLINS y GENTNER, 1987; GENTNER y GENTNER, 1983). Más allá de la diversidad conceptual atribuida al constructo "modelo mental" (GUTIERREZ, 2005), la concepción compartida por todos ellos es que los modelos mentales son representaciones que permiten a las personas interactuar con el mundo, posibilitándoles explicar y predecir el comportamiento de un estado de cosas. Se trata de representaciones idiosincrásicas que se construyen en la memoria de trabajo ante cada situación.

Los modelos mentales han sido mayoritariamente propuestos para interpretar las ideas de los alumnos acerca de algún concepto o fenómeno (modelos mentales de la tierra, modelos mentales del ciclo del día y la noche, modelos mentales de calor, modelos mentales de evaporación, modelos mentales de electricidad, modelos mentales de campo electromagnético, modelos mentales de magnetismo, modelos mentales de la ley de Gauss, modelos mentales de fuerza, modelos mentales de átomo). Estos trabajos no están dirigidos a explicar cómo se construyen y modifican los modelos mentales en términos de la información disponible, sino que tienen por objetivo identificar (o "detectar" siguiendo el lenguaje de algunos autores) los modelos utilizados por las personas cuando razonan acerca de algún fenómeno o concepto físico. Más allá del valor descriptivo de estos estudios, no aparece en ellos, al menos de manera explícita, el lugar de los procesos que actúan sobre los modelos mentales en la interpretación de los registros. Sólo en algunos de estos estudios los objetivos van más allá de la identificación de modelos mentales, cumpliendo éstos un rol secundario, en el sentido que son constructos definidos en el marco de una teoría más abarcativa, según la cual, éstos median el proceso de adquisición de conocimiento de las personas del mundo físico (BUCKLEY y BOULTER, 2000; DIAKIDOY, VOSNIADOU y HAWKS, 1997; VOSNIADOU, 1994; COLLINS y GENTNER, 1987; GENTNER y GENTNER, 1983). En estos casos el poder predictivo está provisto por las teorías en las que los modelos mentales están incluidos como partes de una propuesta más general.

Los métodos más usuales para recolectar datos son encuestas y entrevistas semiestructuradas conformadas por un conjunto de preguntas relativas a la temática investigada. Estas preguntas están confeccionadas de manera que los sujetos predigan y/o expliquen algún comportamiento cuando se los enfrenta a alguna situación problemática sencilla. Las respuestas definitivamente procesadas son las explicaciones que los sujetos dan de sus predicciones. Las predicciones son únicamente utilizadas para dar lugar a las explicaciones. En general, las respuestas válidas para el análisis son aquellas que siguen de la pregunta del tipo: ¿por qué ocurre? 
Algunos aspectos metodológicos de la investigación...

Se trata de estudios exploratorios dado que las respuestas de los sujetos (explicaciones) a los distintos aspectos del fenómeno investigado, son categorizadas e interpretadas por el investigador a posteriori de la experiencia, identificando cada una de esas categorías a un dado modelo mental. Se trata de "ajustar" las respuestas de los sujetos a modelos pensados por el investigador. En muchos casos se encuentra que las respuestas de los sujetos no se ajustan a un modelo dado, sino a una "mezcla" de ellos, y en otros, que los sujetos mantienen modelos alternativos de acuerdo a la forma de la pregunta planteada, o al lugar que ocupa en el cuestionario o entrevista. En este último caso, las explicaciones que puedan darse estarán directamente relacionadas con la perspectiva teórica utilizada, y en particular, con el grado de presencia de procesos que actúan sobre los modelos mentales. Cuando estos procesos están ausentes, este resultado queda en un plano puramente fenomenológico.

Con respecto al tipo de preguntas planteadas, las explicaciones de los sujetos a sus propias respuestas, han sido objeto de controversia entre algunos psicólogos cognitivos. Cuando los sujetos explican su comportamiento (usualmente requerido ante las preguntas tipo ¿por qué..?, suelen elaborar descripciones elaboradas y coherentes de sus propios procesos mentales, existiendo el riesgo de que esas explicaciones no se correspondan con los verdaderos procesos que dieron origen a su comportamiento. En el caso que los sujetos hayan sido instruidos formalmente, es posible que elaboren una explicación plausible para satisfacer las expectativas del entrevistador, desconociendo o ignorando sus propias creencias al respecto. En otras palabras, es posible que el modelo mental utilizado para predecir no coincida con el modelo mental utilizado para explicar, el que es en última instancia reportado.

Esto significa que tales preguntas (presentes en gran número de estudios empíricos que superan ampliamente a la revisión de este trabajo) debieran ser tomadas con cierta precaución. Los estudios de validez de las encuestas y entrevistas pueden, al menos en principio, detectar la falta de correspondencia entre lo que los sujetos hacen y lo que dicen. Un interesante procedimiento de validez es el utilizado por Vosniadou (1994), a partir de las preguntas denominadas generativas. Las preguntas son pensadas de manera tal que enfrenten a los individuos a situaciones no presentadas en ámbitos escolares y que no contengan la palabra referida al concepto del cual se pretende extraer información. Estas preguntas inducen a los sujetos a generar un modelo mental para responder, evitando que respondan con enunciados memorizados a causa de la instrucción recibida. La relación con los modelos mentales, está dada a partir del test de consistencia provisto por las preguntas durante la entrevista con cada sujeto. El test consiste en determinar para cada entrevistado, si el conjunto de respuestas a todas las cuestiones relativas al concepto investigado, pueden ser explicadas por el uso de un modelo mental genérico.

Por último, algunos estudios sobre modelos mentales son de carácter longitudinal, en el sentido que consideran registros correspondientes a distintos periodos a lo largo de la instrucción. Estos diseños no contemplan, a nuestro criterio, el carácter idiosincrásico de los modelos construidos en la memoria de trabajo. Si estas representaciones son ad-hoc y se construyen ante cada nueva situación en la memoria de trabajo, resulta difícil extraer conclusiones acerca de la evolución temporal de los mismos. 


\section{Síntesis}

La revisión anterior permite identificar algunos alcances y limitaciones en las técnicas para la obtención de datos en investigaciones empíricas acerca de las representaciones mentales construidas por los individuos al realizar una tarea cognitiva. Los estudios de expertos y novatos en resolución de problemas de física utilizan prioritariamente la técnica de pensamiento en voz alta, la cual, como se argumentará en la próxima sección, permite extraer datos válidos para la puesta a prueba de modelos teóricos involucrando representaciones y procesos puestos en juego durante la resolución de problemas de física. Sin embargo, esta técnica se encuentra con importantes dificultades cuando es aplicada a sujetos novatos, reflejadas a menudo en la incompletitud de los protocolos por ellos generados. La indagación directa vía preguntas específicas, utilizada en los estudios de modelos mentales, evita la incompletitud anterior, pero pone en riesgo la validez de dichos registros. En el próximo apartado se presenta una propuesta que, mediante la incorporación de algunos elementos teóricos, permite realizar una evaluación fundamentada para la validez de los datos en la tarea que nos ocupa.

\section{Los protocolos verbales}

Los fenómenos mentales, como muchos otros fenómenos de interés científico, no son accesibles a la observación directa. Sin embargo, la mente humana es un sistema consciente, por lo que tiene relativo acceso a sus procesos mentales. Esta característica hace verosímil pensar en la auto-observación o introspección como una alternativa válida para el estudio de la cognición humana. Las técnicas introspectivas han sido utilizadas desde el comienzo de la psicología experimental hasta la actualidad, aunque bajo diferentes concepciones. No obstante, tanto la introspección clásica como la actual (DE VEGA, 1995), han sido objeto de críticas demoledoras por parte de muchos psicólogos cognitivos. En lo que sigue se intenta analizar en qué medida, y bajo que circunstancias, los reportes verbales generados por los sujetos investigados pueden ser tomados como datos válidos a ser interpretados.

Una de las críticas más representativas a los reportes verbales, es la realizada por Nisbett y Wilson (apud ERICSSON y SIMON, 1993). Allí citan un conjunto de trabajos a partir de los cuales concluyen que la gente, a menudo no puede reportar válidamente los estímulos que dan lugar a las respuestas basadas en inferencias. Encuentran que cuando se les pregunta a los sujetos acerca de sus procesos, más que responder basándose en la información para eventos específicos, ellos teorizan acerca de sus procesos, elaborando hipótesis acerca de las causas de sus comportamientos dando respuestas plausibles. En otras palabras, que las personas normalmente no pueden reportar la existencia del estímulo, ni la existencia de la respuesta, ni del proceso inferencial intermedio.

En respuesta a las críticas anteriores, Ericsson y Simon (1993) proponen analizar la validez de los reportes verbales como fuentes de datos, a partir de un modelo cognitivo basado en representaciones y procesos actuando sobre ellas. La propuesta metodológica, "adjunta" un modelo teórico para la memoria que es suficientemente general, consistente con numerosas propuestas actuales del funcionamiento cognitivo, y en función del cual delimitan los alcances y las limitaciones de los protocolos verbales como fuentes de información. 
Algunos aspectos metodológicos de la investigación...

La idea general consiste en considerar que un proceso cognitivo puede ser visto como una secuencia de estados internos que son sucesivamente transformados a medida que la información es procesada. Más específicamente, la información se almacena en diferentes memorias con diferentes capacidades y características para su recuperación: las memorias sensoriales que son de muy corta duración, una memoria de corto plazo (MCP) con capacidad limitada y duración intermedia, y una memoria de largo plazo (MLP) de mayor capacidad y estabilidad en el tiempo, pero de fijación y accesibilidad lentas si se la compara con las dos anteriores. Bajo esta hipótesis, se asume que la información recientemente adquirida por el procesador central, es mantenida en la MCP (es decir atendida) y directamente accesible para posteriores procesamientos (por ejemplo para producir reportes verbales). Por su parte, la información proveniente de la MLP, debe ser primero recuperada (mantenida en la MCP) a fin de ser reportada. Dada la capacidad limitada de la MCP, sólo la información recientemente mantenida es directamente accesible. Sin embargo, parte de los contenidos de la MCP pueden ser fijados en la MLP antes de ser perdidos, posibilitando su recuperación posterior.

En síntesis, el proceso de pensamiento, puede ser descripto como una secuencia de estados, cada uno de los cuales conteniendo el producto final de un proceso cognitivo. La información contenida en esos estados puede ser información recuperada de la MLP, información percibida y reconocida, e información generada por inferencias. La información en cada estado es relativamente estable y puede ser el punto de partida para un proceso de verbalización. Sin embargo, los procesos de recuperación y reconocimiento que llevan sus productos finales a la atención, no pueden ser reportados. Con este modelo en mente, se analizan las condiciones de validez de los protocolos verbales generados durante la realización de una tarea cognitiva o inmediatamente después de haberla concluido.

\section{Protocolos verbales concurrentes o pensamiento en voz alta}

Se trata de protocolos generados durante la realización de una tarea. La instrucción usual es pedir a los sujetos que "piensen en voz alta". No se solicita que describan o expliquen qué están haciendo. En estas condiciones, ellos verbalizan la información mantenida en la MCP mientras están generando la respuesta, sin alterar la secuencia de sus pensamientos. Sin embargo, si a los sujetos también les pide que describan o expliquen sus pensamientos, éstos acceden a información y pensamientos adicionales (que no son los mantenidos en su MCP durante la realización de la tarea) en orden de responder a tal requerimiento. Como resultado, se altera la secuencia de pensamientos, ya que los sujetos deben dirigir su atención a información que no ha sido la necesaria para realizar la tarea.

Dado que las descripciones, justificaciones y explicaciones, son verbalizaciones de origen social y utilizadas como el principal medio de comunicación con otras personas, es usual que los sujetos interpreten la consigna de pensar en voz alta como un pedido de dar una descripción, justificación, o explicación de lo que ellos están haciendo. Para evitar falsas interpretaciones, es conveniente aclarar antes de comenzar la experiencia, que no es una interacción social lo que se pretende establecer entre el investigador y el sujeto. Acorde a esto, se debe intentar minimizar la presencia del investigador. Una forma de hacerlo es cuidando las expresiones mediante las cuales se pide al sujeto que continúe con la verbalización. Por ejemplo, decir 
"siga hablando" es muy diferente a decir "¿puede decirme qué está pensando ahora?”, ya que la segunda de las alternativas puede ser interpretada como para dar una explicación al experimentador. Otra práctica que intenta minimizar la interacción sujeto-investigador es advertir a los sujetos a focalizar principalmente su atención en la tarea a ser realizada, dejando la verbalización en segundo plano. Sólo cuando las verbalizaciones están completamente centradas en la tarea, se pueden esperar las mismas secuencias de pensamiento que en la condición de silencio.

La tarea que se presenta al sujeto también es una variable importante. Dada la importancia de focalizar la atención en la tarea, éstas debieran ser suficientemente claras y explícitas. El trabajo de Garner (apud ERICSSON y SIMON, 1993) muestra que cuando se presentan tareas vagas y generales los sujetos parecen redefinir la tarea mas específicamente, y tal proceso está influenciado por extraños factores sociales que alteran el proceso natural de pensamiento que se desea inducir.

\section{Reportes verbales retrospectivos}

Cuando el reporte comienza una vez finalizada la tarea se denomina retrospectivo. La demanda que se plantea usualmente al individuo es que recuerde todo lo posible acerca de sus pensamientos durante la ejecución de la tarea.

De acuerdo al modelo de procesamiento planteado, la secuencia de pensamientos ocurridos durante la realización de una tarea es almacenada en la MLP. Inmediatamente después de que la tarea ha sido finalizada, aún quedan pistas en la MCP que hacen posible la recuperación de esa información desde la MLP. Para tareas que no superan los 10 segundos de duración, es de esperar que los sujetos estén habilitados para recordar la real secuencia de sus pensamientos, constituyendo datos muy precisos y completos. Para tareas muy cortas, menores a unos pocos segundos, los reportes retrospectivos constituyen inclusive mejores datos que los reportes concurrentes, dada la imposibilidad de verbalizar tan rápidamente. Sin embargo, si la tarea es de mayor duración (superando los 10 segundos) el recuerdo se torna difícil y posiblemente incompleto. No obstante, el reporte retrospectivo, junto con el reporte concurrente de la misma tarea puede ser muy útil para obtener mayor información acerca del proceso de pensamiento en términos más generales.

La principal fuente de imprecisiones de los protocolos retrospectivos proviene de la separación temporal entre la ocurrencia de los pensamientos y el reporte de los mismos. La operación de recuperar información desde la MLP es falible y el sujeto puede recuperar equivocadamente estructuras similares de su memoria, que no corresponden a las utilizadas para realizar la tarea. La probabilidad que esto ocurra aumenta si el sujeto ha resuelto una serie de situaciones similares. El proceso de recuperación puede dar como resultado información relacionada con la tarea, pero que fue adquirida previamente a la ejecución de la misma.

Otro resultado indeseable ocurre si a los sujetos se les pide que reporten información que ellos nunca han mantenido en su MCP mientras realizaban la tarea, lo más posible es que generen o infieran una respuesta plausible a partir de información disponible desde la MLP. Las pruebas que más comúnmente dan lugar a esta situación son las preguntas tipo “¿porqué usted hizo eso?". El sujeto puede, en el mejor de los casos, reportar información que mantuvo durante la resolución del problema (reporte retrospectivo), pero él no tiene acceso a las pistas a partir de las cuales esa información fue generada. Tales reportes se denominan expli- 
Algunos aspectos metodológicos de la investigación...

caciones a posteriori y dan lugar a respuestas que son razones plausibles, pero claramente no constituyen un reporte retrospectivo.

\section{Los protocolos verbales y la resolución de problemas de Física en un ámbito de instrucción formal}

Los párrafos anteriores arrojan importantes pautas para tener en cuenta en la recolección de datos a ser interpretados desde algún modelo cognitivo. Sin embargo, existen algunas restricciones para su aplicación si el interés está centrado en la resolución de problemas en un ámbito de instrucción formal.

La primera restricción está relacionada con la utilización de protocolos verbales retrospectivos. Dependiendo del problema y del nivel de experticia del sujeto, el tiempo medio necesario para resolver un problema usual de física supera la extensión temporal recomendada para la generación de reportes verbales retrospectivos fiables (10 segundos). La duración de esta tarea está relacionada con la necesidad de recuperar información de la MLP a fin de operar con ella en la MCP durante la resolución, y condiciona desde nuestro punto de vista, la utilización de protocolos retrospectivos como registro único de la investigación.

Una segunda restricción reside en el número de sujetos involucrados en la investigación. Uno de los objetivos que persigue la investigación educativa es la posibilidad de aplicar los resultados obtenidos a contextos de características similares. La posibilidad de generalización (al menos dentro de ciertos límites), requiere de muestras suficientemente representativas del universo en cuestión, lo que conlleva un número considerable de sujetos participantes de la investigación. En tales circunstancias se hace difícil obtener protocolos verbales individuales, no sólo por el tiempo que lleva registrar esa información, sino fundamentalmente por el tiempo que lleva transcribir los protocolos. Sin embargo, los protocolos verbales correspondientes a un subgrupo reducido de participantes elegido aleatoriamente pueden proveer importante información complementaria de registros escritos del grupo completo.

Una alternativa de compromiso ante muestras numerosas, consiste en reemplazar los protocolos verbales por actividades de lápiz y papel. Este reemplazo es plausible siempre que se incluyan instrucciones de escribir todo lo que piensen mientras resuelvan, incluyendo dificultades e inconsistencias. Hablando en términos de procesos y productos, lo que un sujeto escribe es el resultado de un proceso de pensamiento puesto en juego durante la solución. En todo caso, la desventaja reside en que un protocolo verbal arroja mayor cantidad de tales productos, ya que el sujeto escribe menos de lo que verbalizaría en un protocolo. Se trata de un compromiso entre validez externa (posibilidad de generalización en algún contexto) y densidad de datos registrados.

Por último, una restricción no menos importante es la capacidad natural de pensar en voz alta. No todas las personas pueden verbalizar naturalmente sus pensamientos. Esta característica, que se acentúa cuando los sujetos son novatos en un dominio específico de conocimiento, puede minimizarse entrenando previamente a los sujetos a pensar en voz alta mientras realizan tareas similares. Mientras mayor sea la familiaridad con la técnica de pensar en voz alta, menor es la necesidad del investigador a intervenir en el proceso de verbalización para alentar al sujeto a continuar hablando, optimizando así la validez de los datos recogidos. 


\section{A modo de conclusiones}

La revisión de los estudios involucrados en este trabajo muestra que los protocolos verbales y las entrevistas semiestructuradas son los métodos más usuales para recolectar información cuando se investigan las representaciones internas de sujetos ante tareas cognitivas. Se han presentado algunas características de estas técnicas, intentando destacar bondades y limitaciones en contextos de interés para la investigación educativa. En particular parece conveniente "respetar" la naturaleza de la representación propuesta por el investigador, para luego seleccionar técnicas adecuadas para su observación. Si se trata de una representación dinámica sobre la que actúan ciertos procesos, una entrevista semiestructurada podría favorecer sesgos e interferencias no deseadas en las respuestas de los sujetos. A menos que las preguntas hayan sido especialmente seleccionadas y validadas en el contexto de la representación investigada, los protocolos verbales, convenientemente implementados e interpretados, parecerían ser los candidatos más adecuados para el estudio de tales representaciones.

Tal es el caso de la resolución de problemas en física, donde los procesos que actúan sobre las representaciones dotan a la representación de un carácter dinámico. Conforme a esta concepción, y a partir de un modelo elemental de memoria, se han argumentado ciertas condiciones para la validez de estos reportes tomados como datos. En particular, tanto la diferenciación entre procesos y productos de la verbalización, como la consideración del contexto social involucrado en esta técnica, orienta la práctica de la misma y la interpretación de los datos recogidos.

En términos más generales consideramos que la utilidad de este trabajo reside, desde nuestra perspectiva, en que brinda algunas herramientas para valorar el alcance de algunos resultados empíricos. La posibilidad de analizar los aspectos metodológicos, no sólo contribuye a la valoración de los resultados, sino que permite comparar resultados que comparten el mismo objeto de estudio. La posibilidad de comparar estudios permite el crecimiento en una línea de investigación. En este sentido, futuros trabajos más completos y representativos en esta dirección podrían arrojar implicaciones tanto para la investigación en este ámbito, como para aquellos lectores y usuarios (usualmente profesores de física) de tales investigaciones. 
Algunos aspectos metodológicos de la investigación...

\section{Referências}

BORGES, T. Models of magnetism. International Journal of Science Education, Londres, v. 20, n. 3, p. 361-378, 1998.

BUCKLEY, B.; BOULTER, B. Investigating the role of representations and expressed models in building mental models. In: GILBERT, J.; BOULTER, C. (Eds). Developing models in Science Education. Dordretcht: Kluwer, 2000. p. 119-135.

CHI, M. T. H.; FELTOVICH, P. J.; GLASER, R. Categorization and representation of Physics problems by experts and novices. Cognitive Science, Norwood, Nova Jersey, n. 5, p. 121-152, 1981.

CLEMENT, J. Analysis of clinical interviews: foundations and model viability. In: KELLY, A.; LESH, R. (Eds.). Handbook of Research Design in Mathematics and Science Education. London: Lawrence Erlbaum Associates, 2000. p. 547-589.

COLLINS, A.; GENTNER, D. How people construct mental models. In: HOLLAND, D.; QUINN, N. (Eds.). Cultural models in language and thought. Cambridge: Cambridge University Press, 1987. p. 243-265.

DE VEGA, M. Introducción a la psicología cognitiva. Madrid: Alianza Psicología, 1995.

DIAKIDOY, I.; VOSNIADOU, S.; HAWKS, J. Conceptual change in Astronomy: models of the earth and of the day/night cycle in American-Indian children. European Journal of Psychology of Education, Lisboa, v. 12, n. 2, p. 159-184, 1997.

ERICSSON, A.; SIMON, H. Sources of evidence on cognition: a historical overview. In: MERLUZZI, T. V.; GLASS, C. R.; GENEST, M. (Eds.). Cognitive assessment. New York: The Guilford Press, 1981. p. 16-51.

ERICSSON, A.; SIMON, H. Protocol analysis. Cambridge: MIT Press, 1993.

GENTNER, D.; GENTNER, D. R. Flowing waters or teeming crowds: mental models of electricity. In: GENTNER, D.; STEVENS, A. (Eds.). Mental models. Hillsdale: Lawrence Erlbaum Associates, 1983. p. 99-129.

GRECA, I.; MALLMANN, L. Modelos mentais do conceito de força. In: ENCONTRO NACIONAL DE PESQUISA EM ENSINO DE CIENCIAS, 1., 1997, Porto Alegre.

Atas... Porto Alegre: Instituto de Física da UFRGS, 1997. p. 280-93.

.; MOREIRA, M. The kinds of mental representations - models, propositions and images - used by college Physics students regarding the concept of field. International Journal of Science Education, Londres, v. 19, n. 6, p. 711-724, 1997.

GUTIERREZ, R. Polisemia actual del concepto "Modelo Mental". Consecuencias para la investigación Didáctica. Investigações em Ensino de Ciências, Porto Alegre, v. 10, n. 2, 2005. Disponível em: < http://www.if.ufrgs.br/public/ensino/revista.htm>. Acesso em: 20 out 2006. 
Buteler, L.; Gangoso, Z.

.; OGBORN, J. A causal framework for analysing alternative conceptions.

International Journal of Science Education, Londres, v. 14, n. 2, p. 201-220, 1992.

HALLOUN, I. Schematic modeling for meanigfull learning of Physics. Journal of Research in Science Teaching, Nova York, v. 33, n. 9, p. 1019-1041, 1996.

HARRISON, A.; TREAGUST, D. Secundary student's mental models of atoms and molecules: implications for teaching Chemistry. Science Education, Salem, Massachussets, v. 80, n. 5, p. 509-534, 1996.

KRAPAS, S.; ALVES, F.; CARVALHO, L. R. Modelos mentais e a lei de Gauss. Investigações em Ensino de Ciências, Porto Alegre, v. 5, n. 1, p. 7-21, 2000.

LARKIN, J. The role of problem representations in Physics. In: GENTNER, D.; STEVENS, A. L. (Eds.). Mental models. Hillsdale: Lawrence Erlbaum Associates, 1983. p. $75-98$.

POZO, J. I. Teorías cognitivas del aprendizaje. España: Ediciones Morata, 1989.

TABER, K. Mediating mental models of metals: acknowledging the priority of the learner's prior learning. Science Education, Salem, Massachussets, v. 87, n. 7, p. 732-758, 2003.

THAGARD, P. Mind. Cambridge: MIT Press, 1996.

TREAGUST, D.; CHITTLEBOROUGH, G.; MAMIALA, T. Students understanding of the descriptive and predictive nature of teaching models in inorganic Chemistry.

Research in Science Education, Holanda, v. 34, n. 1, p. 1-20, 2004.

VOSNIADOU, S. Capturing and modeling the process of conceptual change.

Learning and Instruction, Oxford, Inglaterra, n. 4, p. 45-69, 1994.

Artigo recebido em dezembro de 2006 e aceito em junho de 2007. 